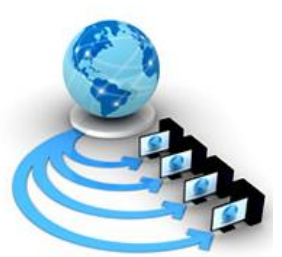

Volume 8, No. 9, November-December 2017

International Journal of Advanced Research in Computer Science

RESEARCH PAPER

Available Online at www.ijarcs.info

\title{
BAYESIAN NETWORKS FOR DETECTION OF VEHICLES UTILIZING PIXEL INSIGHTFUL GROUPING APPROACH
}

\author{
Yerrolla Chanti \\ Assistant. Professor, \\ Department of Computer Science \& Engineering, \\ SR Engineering College, \\ Ananthasagar, Warangal Urban, \\ Telangana, India.
}

\author{
Sallauddin Mohmmad \\ Assistant. Professor, \\ Department of Computer Science \& Engineering \\ SR Engineering College, \\ Ananthasagar, Warangal Urban, \\ Telangana, India
}

\author{
G.Sunil \\ Assistant. Professor, \\ Department of Computer Science \& Engineering \\ SR Engineering College, \\ Ananthasagar, Warangal Urban, \\ Telangana, India.
}

\begin{abstract}
In this paper we proposed an Automatic Vehicle identification framework For Aerial observation. In this framework, a pixel saliently transcription approach for vehicle location is proposed. From the survivable system of finite designation in flying reconnoiter; orders in illumination of locale and sliding window are gotten away. Since the principle drawback is that a vehicle has a tendency to be isolated as various districts when Used to different chromaticity, besides every one of the vehicles are may be gathered as single area in the event that they are comparable .To leave this issue, a Dynamic Bayesian Network (DBN) for vehicle recognition in flying reconnaissance is executed, which depends on the pixel-wise arrangement approach. This pixel-wise grouping protected with the trademark mining process. The highlights are enclosed with vehicle blending and neighborhood the highlights. Hence execution these highlights a Dynamic Bayesian Network (DBN) is worked for the stereotype reason, it changes the local nearby highlights into quantitative location. This investigation is went with a few elevated recordings and the created system is testing the issue with aeronautical observation pictures taken at different statures under the unique point of camera.
\end{abstract}

Keywords- saliently, blending, Dynamic Bayesian Network.

\section{INTRODUCTION}

Aeronautical observation framework has a long history in the military for distinguishing [1] adversary activities and in the beneficial world for watching assets in [2] like timberlands and yields. Alike imaging approaches are utilized as a part of elevated news cast gathering and pursuit and protect aeronautical reconnaissance has been performed at first utilizing film or electronic surrounding cameras. The primary objective is to gathering high determination still pictures of a region underneath reconnaissance that could after be tried by human or machine experts to create data of intrigue. Directly, there is expanding interest in utilizing camcorders for these sorts of assignments. Video takes the dynamic activities that are not comprehended from airborne still pictures. It licenses input and activating of exercises in light of dynamic activities and offers basic and convenient knowledge and understanding that isn't at that point introduced. Video discoveries are utilized to see and geofind the items that are movement progressively and to control the camera, for example, keeping in mind the end goal to take after distinguished vehicles or consistently screen a site. Be that as it may, video additionally conveys new specialized difficulties. Camcorders have lesser determination than those of surrounding cameras, so as to get the determination that is fundamental to identify protests on the ground, it is generally mandatory to utilize a zooming focal point, with a tight field of the view. This watches out for the best genuine weakness of video in observation it offers just a pop straw\| recognition of the scene. The camera should then be filtered to shield areas that are stretched out of intrigue. An onlooker seeing this video should give careful consideration, as objects of interest move rapidly all through the camera field of the perception. The video likewise deficiencies a more visual setting he observer has issue watching the relative places of articles seen at one position so as to objects seen minutes sooner. Moreover, geodetic directions for objects of interest found in the video don't exist. In this paper, another vehicle location outline work is produced in [5] that monitors the advantages of the present works and escapes their inconveniences. The system can be isolated into the accompanying preparing stage and the location stages. Amid preparing stage, we mine numerous qualities containing neighborhood edge and corner attributes, alongside vehicle hues to prepare a dynamic Bayesian system (DBN). Thus, the mined highlights incorporate the data at pixels as well as relationship among nearby pixels in the district. Such outline is more successful and proficient than locale based or multiscale sliding window location strategies.

\section{RELATED WORK}

One of the real subjects in airborne picture examination is scene enlistment [3] and introduction. The examination is begun from issue of picture catch airborne helicopter video for approximating activity parameters and the helicopter check the video precarious, it Is difficult to see and the removed parameters less exact. To influence it to rectify, a casing by-outline video-enrollment approach utilizing a component tracker to over and over direct control-point correspondences is safeguarded. This changes the spatial- 
transient video into the fleeting data, subsequently redressing for airborne stage movement and state of mind botches. The enlistment is solid, with their mining jitter being lesser than constrained pixels over many edges. A simple vehicle discovery conspire discovers vehicle positions in the video, which are then trailed by the element tracker, allowing us to assess the normal speed, moment speed, and remaining parameters consequently to encompass by $10 \%$ of manual counts. The entire technique of enlistment, location, following, and estimation needs just a lesser seconds for each casing. An example interactive media geographic data framework (GIS) is created as a representation apparatus for watching the enrolled video, rest of airborne or satellite symbolism, and information identifying with geo-referenced positions inside a base guide.

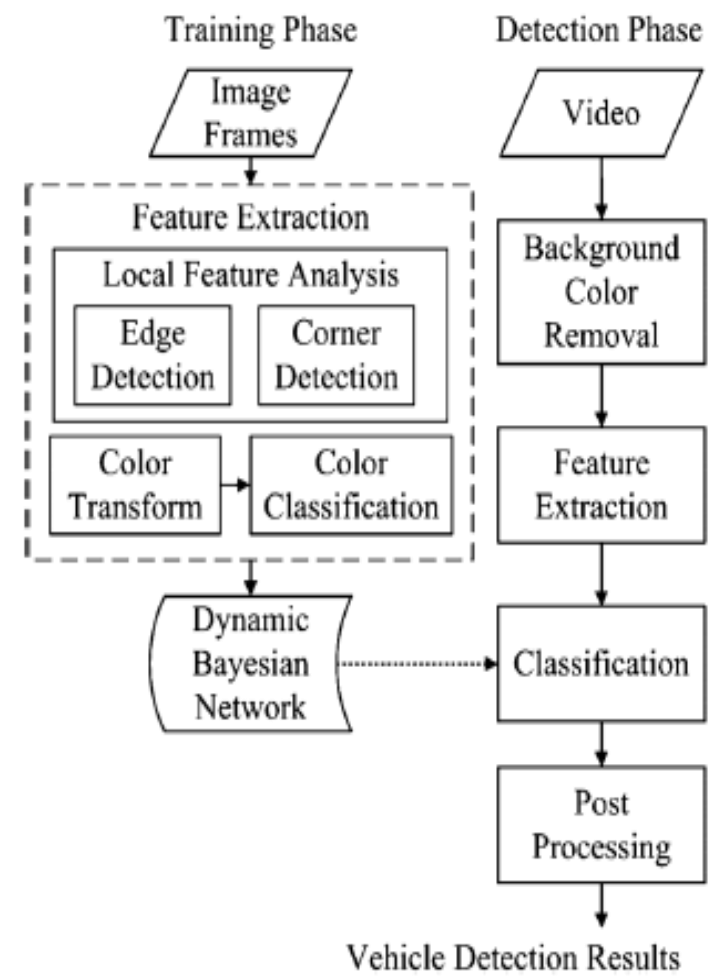

Fig1. Framework Architecture

The other valuable theme in insightful elevated reconnaissance [4] is vehicle Identification and Tracking. The test of vehicle recognizable proof in aeronautical observation encases camera developments like panning, tilting, and pivot. Moreover, airborne stages at a few statures result in different sizes of target objects. The versatile ROI estimation calculation initially researches how the camera is utilized, similar to dish, tilt and zoom control parameters, from the movement computed from the elevated observation video. Utilizing an edge based camera administrator consideration show; it cannot just inexact the ROI of the camera administrator at the edge level, yet additionally estimated the ROI at the arrangement level. Comes about subsequent to directing examinations utilizing video taken by Predator Unmanned Aerial Vehicle UAV) pictures that the proposed versatile ROI estimation calculation is powerful and in the meantime proficient. A standout amongst the most valuable theme is progressive modular that outlines different levels of particulars of vehicle highlights [5]. There is no particular vehicle models gathered, make the approach adaptable. The mining depends on progressive model that assigns the unmistakable vehicle attributes at different levels of detail. Logical learning, Relations between a vehicle and remaining items. These consider various indications and utilize Mixture of Experts district [6] and protest division calculation for airborne observation video. In this paper, we propose most recent vehicle location structures that jelly the advantages of the works that are as of now existed and gets away from their impediments. The modules of the proposed framework structure are portrayed in aSbove Fig. The structure can be isolated into the accompanying preparing stage and the location stage. In the preparation stage, we mine numerous highlights containing neighborhood edge and corner highlights, and also vehicle hues to instruct a dynamic Bayesian system (DBN). In the identification stage, we initially entire foundation shading subtraction comparable to the technique proposed in [9]. At that point, a similar element mining method is connected like the preparation stage. The mined highlights gave as the affirmation to construe the unidentified condition of the prepared DBN, which thusly signifies whether the pixels have a place with the vehicle or not. In this paper, we don't have any significant bearing characterization in light of areas, which would to a great degree rely upon results of shading division calculations like mean move. There is need to deliver multi scale sliding windows. The separating highlight of the proposed setting is that the recognizable proof undertaking depends on the pixel astute classification. However, the highlights are mined in a neighboring locale of each pixel. In this way, the mined highlights incorporate the data at pixel-level as well as connections among nearby pixels in an area. Such plan is also compelling and proficient than the area based [8] or multi scale sliding window recognizable proof techniques [11]. Whatever is left of this paper is organized as takes after: Section IV give subtle elements of the modules of the proposed programmed vehicle location component in detail. Area $\mathrm{V}$ expresses and investigates the test comes about. Finally, conclusions are made in Section VI

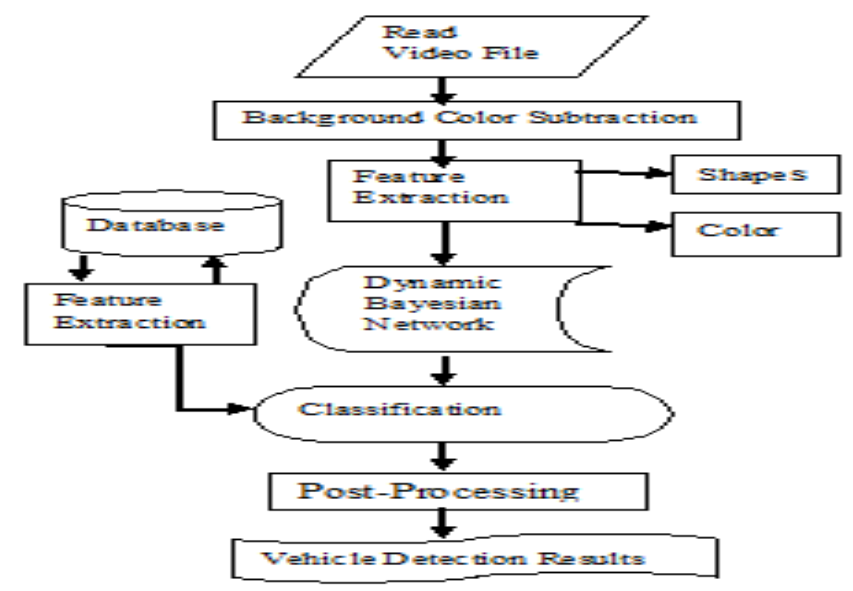

Vehicle Detection Using Pixel shrewd Classification Approach

Fig 2: Propose framework

3.PIXEL INSIGHTFUL PROCESS 


\section{Foundation Color Subtraction:}

Foundation Color subtraction is one of the great strategies of the picture preparing. The back ground is evacuated in any pictures simple to distinguish protests in reality. In this paper is utilization to this method gives better recognizable proof of vehicles in discovery stages. This strategy is connected in whole locales of the system in movement control frameworks and it will be utilized as a part of shading histogram calculation. The calculation is gives whole area of system gives shading in containers shrewd[11]. On the off chance that you evacuate the shading in every now and again in subtraction of foundation you can without much of a stretch distinguish vehicles and non vehicle locales in this procedure. At that point we apply the shrewd edge location and Harris corner indicator is utilized to play out the reaming operation in vehicle recognition process. It will expand the speed discovery process when the fruition of back ground shading subtraction process.

\section{Shading Histogram Algorithm:}

Read video document database and concentrate RGB organize pixel data from pictures. Make 48 canister standardized histograms for each of the RGB segments of each picture read from Database and each picture will have 3 histograms related with it. Shading Histogram most noteworthy receptacles are oftentimes foundation shading evacuated. Identified in pixels won't perform promote resulting identification process. Recognition accelerates increment and false alert decreased.

\section{Highlight Extraction Techniques:}

Highlight extraction is performed both preparing and recognition stages. Highlight extraction considers as neighborhood highlights and additionally shading highlights in this framework. In this strategy we extricate the element from the picture outline. In this technique we do the accompanying Edge Detection, Corner Detection, shading Transformation and shading grouping strategy is utilized to give better outcomes contrasted with existing methodologies.

\section{Neighborhood Feature Analysis:}

The picture contains more data in pixels however every pixel contains corners and edges, so we utilize the Harris corner indicator [8] is utilized to distinguish corners in vehicles. Next we utilize shrewd edge finder [6] is utilized to identify the edges in vehicles. In the edge discovery in view

ofmomentpreservingthresholdingmethodwillbecalculatingdif ferentscenesinaerialimageofvehicleiin the shrewd edge finder, there are two Importance Thresholding. I.e. the lower limit low and the higher edge is $\mathrm{T}$ high. As brightening in each airborne picture contrasts to the coveted limit fluctuate and versatile edges are required in edge identification stages.

\section{Shading Transform and Classification:}

In this paper proposed new shading change show is to isolate vehicle hues [12] and non-vehicle hues from adequately. This shading changes (R, G, B) shading segments into the shading spaces $(u, v), U p=(2 Z p-G p-$ B p)/Z p Where $(R, G, B)$ is the $R, G$, and $B$ shading segments of pixel $p$ and $Z p=(R p+G p+B p) / 3$. It has been appeared in that all vehicle hues are moved in substantially littler regions on the $\mathrm{u}-\mathrm{v}$ plane than in other shading spaces and are in this manner less demanding to be isolated from non vehicle hues. SVM characterization is utilized to nonvehicle shading ranges distinguished. The extraction procedure is five sorts is $\mathrm{S}, \mathrm{C}, \mathrm{E}, \mathrm{A}, \mathrm{Z}$ for a pixels. These highlights fill in as perceptions to gather the obscure condition of a DBN, which will be expounded in the following segment. The pixels that are delegated vehicle hues are named as associated vehicle shading areas. A, Z are characterized as the perspective proportion and the extent of the associated vehicle shading locale where the pixel P lives. A=Length/Width, $\mathrm{Z}$ = include of pixels "vehicle shading district Color classification is using SVM machine

\section{Arrangement:}

In this approach we perform pixel astute grouping for vehicle location utilizing Dynamic Bayesian Networks $(\mathrm{DBN})$. The DBN is performed in both preparing and identification stages. In the preparation organize, we acquire the restrictive likelihood tables of the DBN demonstrate by means of desire expansion calculation by giving the groundtruth naming of every pixel and its relating watched highlights from a few preparing recordings. In the discovery stage, the Bayesian control is utilized to get the likelihood that a pixel has a place with a vehicle at specific time cut.

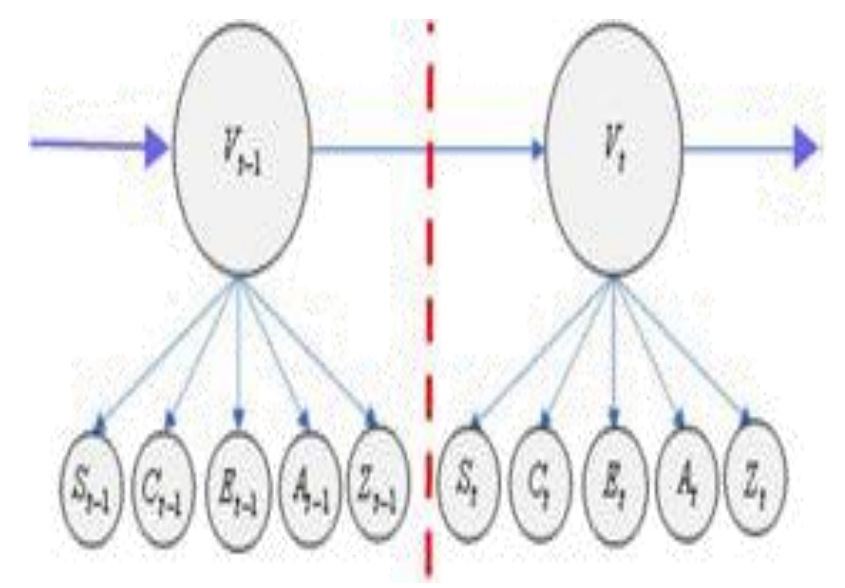

Fig 4: DBN show for Pixel shrewd Classification

The outline of the DBN demonstrate is given fig: 4 , a hub $t$ shows if a pixel has a place with a vehicle at time cut $t$. In the province of $\mathrm{Vt}$ is subject to the territory of Vt-1. At each time cut $t$, state $\mathrm{V} t$ has impacts on the perception hubs St, $\mathrm{Ct}, \mathrm{Et}, \mathrm{A} \mathrm{t}$, and $\mathrm{Z} \mathrm{t}$. The perceptions are not reliant in any others. Discrete perceptions images are utilized as a part of our framework. We utilize k-intends to bunch every perception into three group sorts that are the preparation arrange we gets the restrictive likelihood tables of the DBN show by means of special case augmentation calculation by giving the ground truth naming of every pixel and its relating watched highlights from a few preparing recordings. In recognition arrange the Bayesian govern is utilized to acquire the likelihood that a pixel has a place with a vehicle. Proposed vehicle discovery structure can likewise use a Bayesian Network (BN) to arrange a pixel as a vehicle or 
non vehicle pixel. When performing vehicle recognition utilizing $\mathrm{BN}$, the structure of the $\mathrm{BN}$ is set as one time cut of the DBN show.

\section{Post handling:}

The post handling technique we utilize morphological operations to improve the recognition cover and perform associated part marking to get the vehicle objects. The size and the perspective proportion limitations are connected again after morphological operations in the post handling stage to dispose of articles that are difficult to be vehicles. In any case, the limitations utilized here are free. By utilizing pre-preparing method lessened of the discovery objects look at existing frameworks. On the off chance that any vehicle is absent on the location in beginning stages will be recognized in this stage.

\section{MODULES}

In this framework the vehicle identification depends on consequent modules:

1. Frame Extraction

2. Background Color Removal

3. Highlight Extraction

4. Arrangement

5. Post Processing

Module 1: Frame Extraction In this module.avi video is given as information; at that point complete an edge mining operation on video. Later finishing outline mining different casings are created powerfully and kept up in relating outlines envelope.

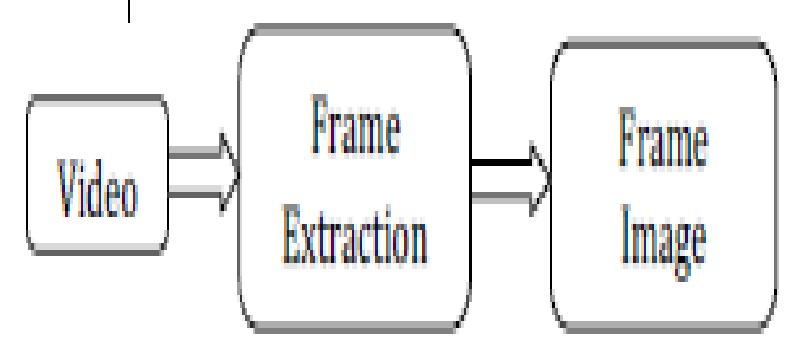

Fig 3.Edge Extraction

Module 2: Background Color Removal In this module dispense with the foundation shading from the edges, apply the shading order for vehicle and non-vehicle different hues, more than once every one of the photos are kept up in foundation outlines Folder.

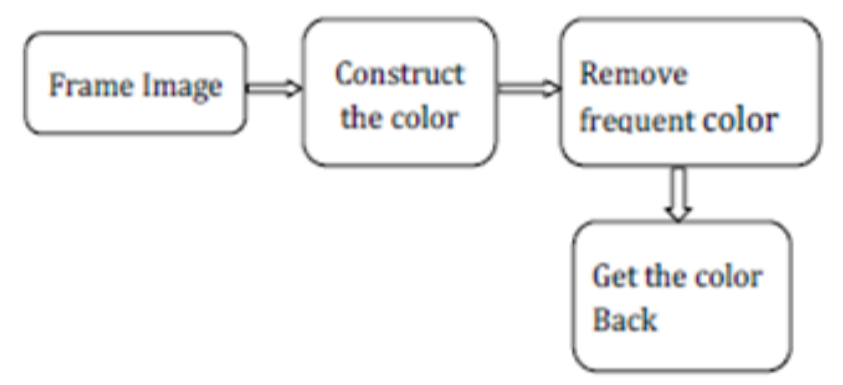

Fig 4.Back ground shading evacuation
Module 3: include extraction In this module take the neighborhood highlights and shading highlights. This module relies upon two modules portrayed previously. Edge Image is proficient to move by finishing recognizing edges, corners and spaces for changing shading and store the outcome in recognition organizer.

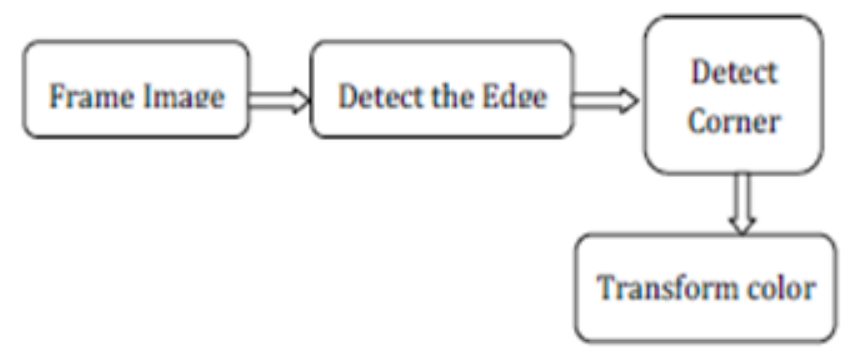

Fig5. Highlight Extraction

Module 4: order In this module finish pixel savvy grouping for vehicle distinguishing proof utilizing DBN technique. In the recognition stage, the Bayesian manage is utilized to procure the likelihood that a pixel have a place with a vehicle.

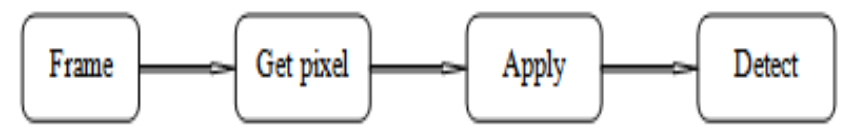

Fig 6.Grouping

Module 5: post preparing In this module morphological activities to enhance the discovery cover and do associated segment naming to get the vehicle objects.

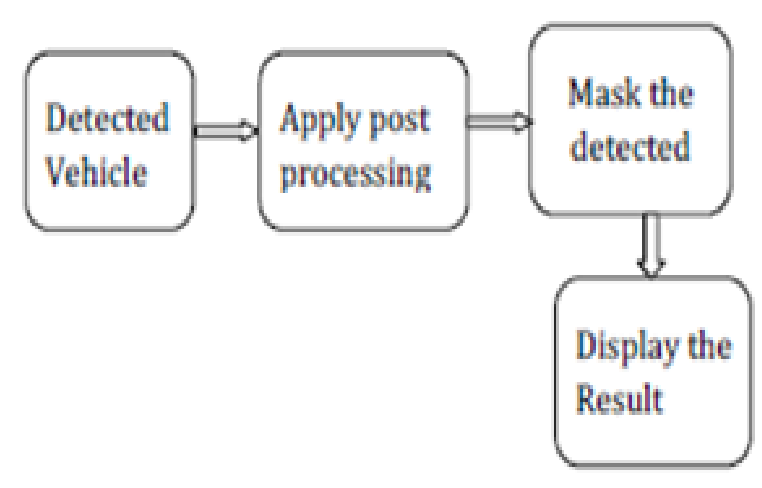

Fig 7.post handling

\section{Calculation:}

The Canny Edge Detection Algorithm: The Canny calculation on a very basic level obtains sedges in which the dark scale force of the photo alters the cost. These ranges are acquired by conclusive inclinations of the photo. Inclinations at each pixel in the round picture are discover by performing what is called as Video the Soberadministrator. Initial step is to assess the inclination in the $\mathrm{x}$ and y-course correspondingly by playing out the portions. The calculation keeps running in 5 singular advances: 
Step.1: Smoothing: Blurring of the picture to take out clamor It is unavoidable that all pictures caught from the camera will incorporate some amount of clamor. To stop that clamor is mixed up for edges, commotion must be diminished.

Step.2: Finding Gradients: The edges ought to be detectable where the slopes of the photo have expansive sizes.

Step.3: Non-Maximum Suppression: Only nearby maxima must be set apart as edges.

Step.4: Double Thresholding: Potential edges are registered by thresholding.

Step.5: Edge Tracking by Hysteresis: Last edges are discovering by containing every one of the edges that are not joined to an extremely specific edge

\section{RESULT ANALYSIS:}

The examination of the proposed framework and its execution can be depicted in this segment. The evaluation is starting with a few vehicle discovery techniques[13]. The moving vehicle identification with street recognition technique needs setting of part more parameters to force the size confinements so as to diminish false cautions. The examination and execution is in advance with the different situations, for example, without expelling foundation, without upgraded edge indicator, without foundation expulsion and with improved edge identifier. The execution is tried with the before recognition procedures and furthermore with our proposed DBN. The ensuing chart pictures the execution advancement between various vehicle recognition methods.

\begin{tabular}{|c|c|c|}
\hline Method & Hit ratio & $\begin{array}{c}\text { Number of false } \\
\text { positives per frame }\end{array}$ \\
\hline MVDRD & 72.09 & 0.499 \\
\hline Symmetnc properties & 74.96 & 0.450 \\
\hline Cascadec classifier & 78.08 & 0399 \\
\hline Proposed BN & 92.31 & 0.297 \\
\hline Proposed DBN & 9235 & 0.278 \\
\hline
\end{tabular}

Fig 8.table of result investigation

In this, the appraisal examination gave the hit proportion and number of false positives per outline. Here the DBN out performs BN. While watching recognizable proof aftereffects of progressive edges, the discovery comes about by means of DBN are more consistent. The reason is that in ethereal observation the flying machine moving the camera viably following vehicles on the ground, and subsequently the areas of the vehicles would not have dramatic adjustments in the scene notwithstanding when the vehicles are moving with substantial rates.

Presently the examination is begins with the paces of various discovery procedures. The consequent outline imagines the normal preparing rates of different vehicle location strategies. The trials are performed on a PC. The proposed system utilizing DBN and BN can't achieve the casing rate of the reconnaissance recordings; it is sufficient to finish vehicle recognizable proof of each 50 to hundred edges.

\section{CONCLUSION}

In this paper, we have proposed a programmed vehicle location framework for elevated observation that does not expect any earlier data of camera statures, vehicle sizes, and viewpoint proportions. In this framework, we have not performed area based arrangement, which would profoundly rely upon computational serious shading division calculations, for example, mean move. We have not created multi scale sliding windows that are not reasonable for distinguishing pivoted vehicles either. Rather, we have proposed a pixel insightful arrangement technique for the vehicle location utilizing DBNs. Despite Performing Pixel astute order, relations among neighboring pixels in a district are safeguarded in the element extraction process. Hence, the removed highlights involve pixel-level data as well as area level data. Since the shades of the vehicles would not significantly change because of the impact of the camera edges and statures, we utilize just few positive and negative examples to prepare the SVM for vehicle shading characterization. In addition, the quantity of casings required to prepare the DBN is little. By and large, the whole system does not require a lot of preparing tests. We have additionally connected minute saving to upgrade the canny edge indicator, which expands the flexibility and the precision for identification in different elevated pictures. The exploratory outcomes show adaptability and great speculation capacities of the proposed technique on a testing informational index with airborne observation pictures taken at various statures and under various camera edges. For future work, Performing vehicle following on the identified vehicles can additionally balance out the location comes about. Programmed vehicle recognition and following could fill in as the establishment for occasion examination in astute elevated observation frameworks

\section{ACKNOWLEDGMENT}

Authors would like to express sincere gratitude to management and principal of S R Engineering College, for their support and encouragement to carry out the research work.

\section{REFERENCES}

[1] R. Kumar, H. Sawhney, S. Samarasekera, S. Hsu, T. Hai, G.Yanlin, K. Hanna, A. Pope, R. Wildes, D. Hirvonen,M. Hansen, and P. Burt, -Aerial video reconnaissance and exploitation,॥ Proc. IEEE, vol. 89, no. 10, pp. 1518-1539, 2001.

[2] I. Emst, S. Sujew, K. U. Thiessenhusen,M. Hetscher, S. Rabmann, and M. Ruhe, -LUMOS-Airbome movement checking system,\| in Proc. IEEE Intell. Transp. Syst., Oct. 2003. vol. 1, pp. 753- 759.

[3] L. D. Chou, J. Y. Yang, Y. C. Hsieh, D. C. Chang, anC. F. Tung, Intersection-based steering convention for VANETs,\|Wirel. Pers. Commun., vol. 60, no. 1, pp. 105124. Sep. 2011.

[4] S. Srinivasan, H. Latchman, J. Shea, T. Wong, and J. McNair, - Airborne movement observation frameworks: Video reconnaissance of expressway traffic, $\|$ in Proc. ACM second Int. Workshop Video Surveillance Sens. Netw., 2004, pp. 131- 135. 
[5] A. C. Shastry and R. A. Schowengerdt, - Airborne video enrollment and movement stream parameter estimation,\| IEEE Trans. Intell. Transp. Syst., vol. 6, no. 4, pp. 391405, Dec. 2005.

[6] h. Cheng and J.Wus, -Adaptive locale of intrigue estimation for airborne observation video,\| in Proc. IEEE Int. Conf. Picture Process., 2005, vol. 3, pp. 860- 863

[7] S. Hinz and A. Baumgartner, - Vehicle location in airborne pictures utilizing non specific highlights, gathering, and context,\| in Proc. DAGM-Symp., Sep. 2001, vol. 2191, Lecture Notes in Computer Science, pp. 45- 52.

[8] H. Cheng and D. Steward, - Segmentation of flying reconnaissance video utilizing a blend of experts, $\|$ in Proc. IEEE Digit. Imaging Comput. - Tech. Appl., 2005, p. 66.

[9] R. Lin, X. Cao, Y. Xu, C.Wu, and H. Qiao, -Airborne moving vehicle recognition for urban activity surveillance, $\|$ in Proc. eleventh Int. IEEE Conf. Intell. Transp. Syst., Oct. 2008, pp. 163- 167.

[10] L. Hong, Y. Ruan, W. Li, D. Wicker, and J. Layne, —Energy-based video following utilizing joint target thickness handling with an application to unmanned flying vehicle surveillance,\| IET Comput. Vis., vol. 2, no. 1, pp. 1- 12, 2008.

[11] Sallauddin Mohmmad, Shabana , Ranganath Kanakam, Provisioning Elasticity On IoT's Data In Shared-Nothing Nodes , International Journal of Pure and Applied Mathematics, Volume 117 No. 7, pp. 165-173, oct. 2017.

[12] Sallauddin Mohmmad, G. Sunil, Ranganath Kanakam ,A Survey On New Approaches Of Internet Of Things Data Mining, International Journal of Advanced Research in Computer Science, Volume 8, No. 8, September-October 2017 .

[13] G. Sunil , Sallauddin Mohmmad , Kanegonda Ravichythanya, The Current Status And Research In Industrial Big Data Analysis In Smart Intelligent Systems, International Journal of Advanced Research in Computer Science, Volume 8, No. 8, September-October 2017. 